\section{Morphologic and immunophe- notypic features of a case of acute monoblastic leukemia with unusual positivity for Glycophorin-A}

\author{
Giovanni Carulli, ${ }^{1}$ Paola Sammuri, ${ }^{1}$ \\ Cristiana Domenichini, ${ }^{1}$ \\ Martina Rousseau, ${ }^{1}$ \\ Virginia Ottaviano, ${ }^{1}$ \\ Maria Immacolata Ferreri, ${ }^{2}$ \\ Antonio Azzarà, 1 \\ Francesco Caracciolo, ${ }^{1}$ Mario Petrini ${ }^{1}$ \\ ${ }^{1}$ Division of Hematology, University of \\ Pisa; ${ }^{2}$ Laboratory of Medical Genetics, \\ AOUP, Pisa, Italy
}

\begin{abstract}
Acute monoblastic leukemia $(\mathrm{AMoL})$ is characterized by cells with highly undifferentiated morphology. Cytochemistry with non-specific esterases is negative in up to $20 \%$ of cases. Immunophenotyping by flow cytometry has an essential role in diagnosing such a subtype of leukemia and a multiparametric approach with a wide monoclonal antibody panel is necessary. We describe a case of AMoL with morphology resembling either plasma blasts or very immature erythroblasts. Diagnosis was made by alpha-naphtyl-acetate esterase staining and with immunophenotyping, which was made with a wide monoclonal antibody panel. Blasts were positive for monocytic markers. Most of leukemic cells, however, were positive for Glycophorin-A. The presence of Glycophorin-A, which is considered as a specific marker of the erythroid lineage, has never been reported previously in cases of AMoL. This peculiar immunophenotype might be interpreted as deriving from a common myelo-erythroid precursor undergone leukemic transformation.
\end{abstract}

\section{Introduction}

Acute monoblastic/monocytic leukemia (AMoL) is recognized by the 2016 WHO classification as a not specific subtype, NOS (not otherwise specified), of acute leukemias. ${ }^{1}$ According to the FAB (FrenchAmerican-British) classification, AMoL M5a is a subtype characterized by proliferation of monoblasts, which account for at least $80 \%$ of leukemic cells. ${ }^{2,3}$

A morphologic diagnosis of $\mathrm{AMoL}$ is difficult, because monoblasts generally are poorly differentiated cells and can be con- fused with large lymphomatous cells, ${ }^{4}$ or other immature cells, such as plasma blasts, ${ }^{5,6}$ or very immature erythroblasts. ${ }^{7}$

Monoblasts can be defined as large cells (20-30 $\mu \mathrm{m})$, with round/oval nuclear shape, delicate chromatin, prominent nucleolus, basophilic cytoplasm with few azurophilic granules. ${ }^{8}$ Cytochemical staining, such as either alpha-naphtyl-acetate esterase (ANAE) or alpha-naphtyl-butirate esterase (ANBE) can be used to identify monocytes and their neoplastic counterparts. Although more sensitive than ANBE, ANAE can be negative in about $20 \%$ of cases of AMoL. ${ }^{9}$

Flow cytometry has an essential role in the diagnosis and classification of acute leukemia. ${ }^{10}$ Monoblasts are identified by means of $\mathrm{CD}$ (cluster of differentiation) markers which are relatively characteristic of the monocytic lineage. Using multiparameter flow cytometry (MFC) and a broad monoclonal antibody (MoAb) panel, the diagnosis of AMoL can be established in a very high percentage of cases. The most useful CD markers are CD13, CD33, CD15, CD64, CD65s and HLA-DR, 2,9,11,12 which are positive in the vast majority of cases.

In the current paper we describe a peculiar case of AMoL with a very undifferentiated morphology of blast cells, which mimicked plasma blasts or erythroid blasts, and with high percentage of blasts positive for the erythroid markers CD71 and Glycophorin-A. To the best of our knowledge, the positivity of Glycophorin-A in AMoL has not been reported previously.

\section{Case Report}

A Caucasian 41-year-old female, with a previous silent clinical history, complained of fatigue and exertional dyspnea. After a family doctor visit, she carried out a complete blood count $(\mathrm{CBC})$ and chemistries. CBC showed $\mathrm{Hb} 8.5 \mathrm{~g} / \mathrm{dL}$; WBC $8 \times 10^{9} / \mathrm{L}$; PLT $50 \times 10^{9} / \mathrm{L}$. Automated differential of WBC showed an apparent lymphocytosis (60\%), but manual differential, carried out in the Central Laboratory of Clinical Pathology of our Hospital, was consistent with a possible plasma blast leukemia or, in alternative, with a possible derivation of blasts from the erythroid lineage (Figure 1). Chemistries showed very high LDH values $(2,000 \mathrm{U} / \mathrm{L})$. Blood coagulation parameters were normal, as well as the electrophoretic protidogram and immunoglobulin levels. Serum immunofixation did not show any monoclonal component.

The patient was sent to our observation. Ultrasound and TC scans did not show lymphadenomegalies nor hepatosplenomegaly.
Correspondence: Giovanni Carulli, M.D Division of Hematology, Santa Chiara Hospital, Via Roma 67, I-56126 Pisa, Italy. Tel: +39.050992731 - Fax: +39.050993378. E-mail: g.carulli@ao-pisa.toscana.it

Key words: acute monoblastic leukemia, cytomorphology, flow cytometry, glycophorin-A.

Contributions: GC followed the case, was responsible for morphology, supervised immunophenotyping and wrote the manuscript. PS and MR carried out immunophenotyping. CD performed PCR reactions. FC and MP managed the patient. MFI carried out karyotyping. AA was responsible for morphology and cytochemistry. All authors read and approved the manuscript.

Conflict of interest: the authors declare no potential conflict of interest.

Funding: none

Received for publication: 24 August 2018. Accepted for publication: 29 October 2018.

This work is licensed under a Creative Commons Attribution-NonCommercial 4.0 International License (CC BY-NC 4.0).

(C) Copyright G. Carulli et al., 2018

Licensee PAGEPress, Italy

Hematology Reports 2018; 10:7823

doi:10.4081/hr.2018.7823

A myeloaspirate was carried out. Bone marrow aspirate films showed hypercellularity due to massive infiltration by medium-sized to large-sized blasts, with round and often eccentric nucleus, loose chromatin, abundant basophilic cytoplasm and, often, a perinuclear "hof" (Figure 2A). Binuclear cells with erythroblast-like morphology were observed (Figure 2B), as well as occasional cells with cytoplasmic bridges (Figure 2C). Morphology was compatible with: plasma blasts, early erythroid precursors, monoblasts. Myeloperoxidase (MPO; benzidine-based assay) showed few positive cells, while ANAE (diagnostic kit purchased from Sigma-Aldrich, Saint Louis, MO, USA), was strongly positive, with prevalent diffuse pattern, in about $80 \%$ of neoplastic cells (Figure 2D). These results were compatible with AMoL, M5a subtype of FAB classification.

Bone marrow samples were subjected to immunophenotyping by MFC, which was performed by a FacsCanto II cytometer (Becton Dickinson, BD, Palo Alto, CA, USA) equipped with three lasers (405, 488 and $633 \mathrm{~nm}$ ) and assisted by the FacsDiva software (BD). A 6-8 color method was carried out, using MoAbs conjugated with the 
following fluorochromes: FITC, PE, PerCP-Cy5.5, PE-Cy.7, APC, APC-Cy7, Horizon V450, Horizon V-500. All MoAbs were purchased from BD. A lyse-no-wash method was used, and erythrocyte lysing was made by $\mathrm{NH}_{4} \mathrm{Cl}$. Fifty $\mu \mathrm{L} /$ tube of bone marrow samples was incubated with adequate amounts of MoAbs $(7 \mu \mathrm{L}$ for FITC and PE, $5 \mu \mathrm{L}$ for the other MoAbs) for 20 minutes at room temperature in the dark. One hundred thousand events/tube were acquired for the cytofluorimetric analysis.

Blast cells were found in the monocytic area of the CD45/SSC dot-plot. They were positive for CD33, CD15, CD38, CD56, CD64, HLA-DR, CD4 (Figure 3), with a homogeneous pattern of positivity and high fluorescence intensity; low fluorescence intensity was found for CD14 and CD71 (Figure 3C and I), while CD13 was downregulated (Figure 3D). Interestingly, Glycophorin-A was found to be positive in $60 \%$ of blast cells, with high fluorescence intensity (Figure 3I). Blast cells were negative for CD138, and very few events positive for both CD138 and CD38 were found, being classified as residual plasma cells (Figure 3L). Residual lymphocytes were used as internal control and quadrant analysis was performed using lymphocyte fluorescence intensity as a negative control.

Molecular biology assays carried out by polymerase chain reaction (PCR) methods, using the diagnostic kits REALQUALITY RQ-BCR-ABL p210, REALQUALITY RQ-BCR-ABL p210, REALQUALITY RSPML-RARa bcr1, REALQUALITY RSPML-RARa bcr3, REALQUALITY RSAML1-ETO and REALQUALITY RSINV16 (AB Analitica, Padua, Italy) resulted negative for the following fusion-genes: BCR-ABL1, AML1-ETO, PML-RAR $\alpha$, CBF $\beta$-MYH11.

After G-banding technique usage, a karyotype with $47, \mathrm{XX},+8, \operatorname{del}(20 \mathrm{q})$ was detected.

As a result of the above findings, AMoL with aberrant expression of Glycophorin-A was diagnosed.

The patient gave her informed consent to personal data treatment and to diagnostic and therapeutic procedures

\section{Discussion and Conclusions}

A morphologic diagnosis of $\mathrm{AMoL}$ is very difficult, mostly in the case of the M5a subtype of the FAB classification. In this subtype of acute leukemia, blasts share many features with other immature cells, such as plasma blasts and very early erythroblasts. ${ }^{5-7}$ Enzyme cytochemistry is able to detect cells deriving from the monocytic lineage, ANAE being more sensitive than ANBE. ${ }^{9}$ In our case, ANAE was able to identify $\mathrm{AMoL}$, despite the very immature morphology of blast cells.

MFC is the most useful method to detect monoblasts. About $100 \%$ of cases are positive for CD13, CD33, HLA-DR, and
$56-85 \%$ of cases are positive for CD4, CD11b, CD11c, CD14, CD15, CD56, CD64. Other molecules, such as CD34 and CD117 are less frequently expressed by blasts from AMoL (11-45\% of cases). ${ }^{2,9,11-}$

15 Therefore, by using MFC and a wide $\mathrm{MoAb}$ panel, it is possible to identify leukemic monoblasts and diagnose AMoL,

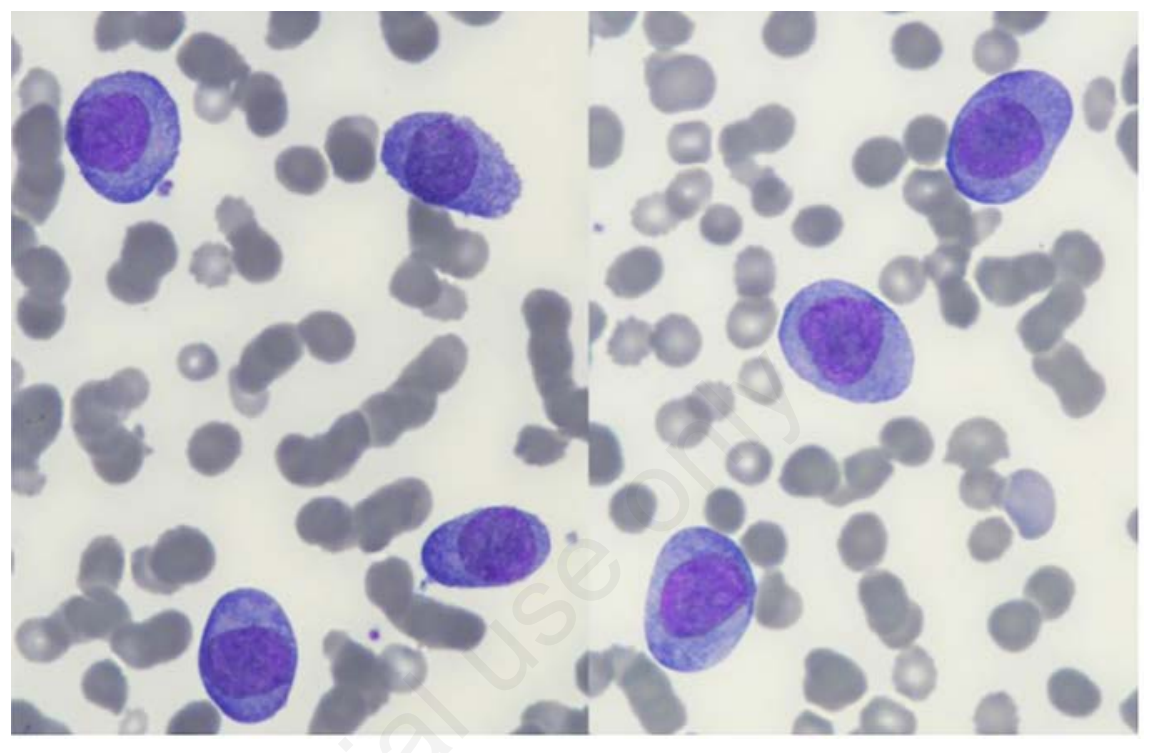

Figure 1. Morphology of circulating blast cells (May-Grünwald-Giemsa; x1,000).


Figure 2. Morphology of myeloaspirate samples. A) massive infiltration by medium- and large-sized blast cells with undifferentiated morphology, often with a perinuclear "hof". B) a binuclear blast with erythroblast-like morphology (arrow). C) two blast cells linked by a cytoplasmic bridge (arrow). D) most of blast cells are positive for ANAE, with a homogeneous pattern of staining; $x 1,000)$. 
A

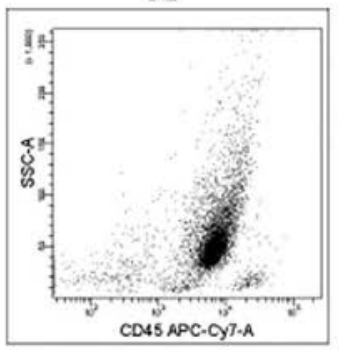

F



B

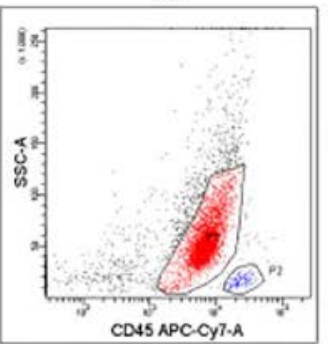

G

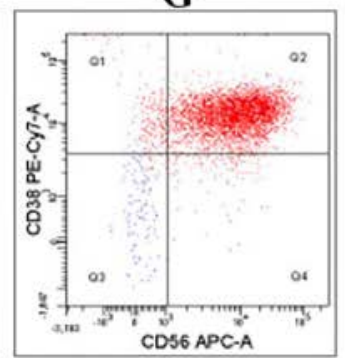

C

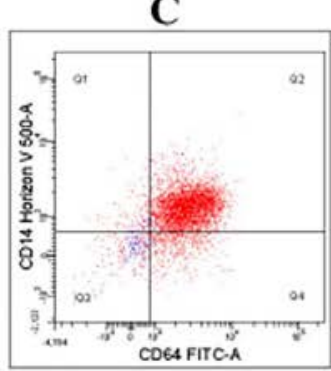

H

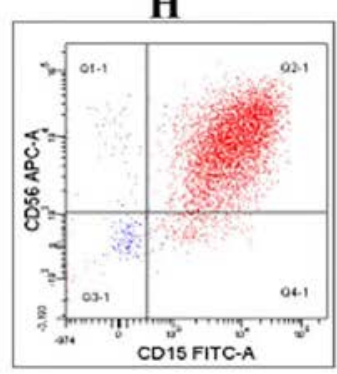

D
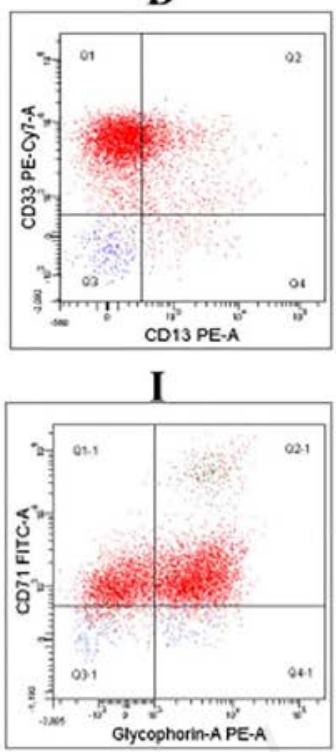

E

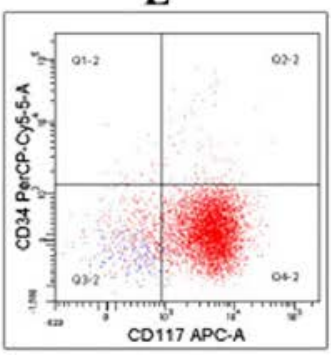

L

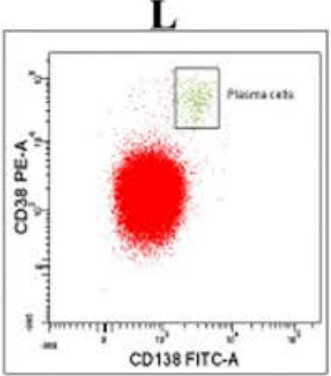

Figure 3. Flow cytometric immunophenotyping of bone marrow, quadrant analysis. A) CD45/SSC properties of blast cells. B) gating of blast cells (P1, red dots) and residual lymphocytes (P2, blue dots). C-H) pattern of expression of monocytic and myeloid markers. I) co-expression of CD71 (with low fluorescence intensity) and Glycophorin-A (which is positive in $60 \%$ of blast cells). L) detection of a very small plasma cell population.

even in cases negative for ANAE and/or ANBE. ${ }^{16}$

In our case, blast cells showed a very undifferentiated morphology and differential diagnoses involved either plasma blast leukemia or erythroid leukemia blasts. However, both cytochemistry and MFC were able to clearly diagnose AMoL.

Interestingly, in our case both CD71 and Glycophorin-A were detected. CD71 is a membranous protein involved in iron uptake and, since most body iron is used by erythroid cells for hemoglobin synthesis, it appears at a very early stage of erythroid development. ${ }^{17}$ This molecule can be used to study the maturation of the erythroid lineage in bone marrow samples: the expression of CD71 is found in pro-erythroblasts and maturating erythroblasts, and is lost when also the nucleus is lost by the orthochromatophilic normoblasts. ${ }^{18}$ CD71, however, is expressed in many cases of acute myeloid leukemia, with low levels of fluorescence intensity in cases of AMoL. ${ }^{19-}$ ${ }^{22}$ Its presence could be related to a high cell proliferative rate. However, some studies have shown that neither citofluorimetric positivity nor the amounts of the specific transferrin mRNA had any correlation with outcome. ${ }^{19,23,24}$

Glycophorin-A (also termed CD235a) is the major glycophorin of human erythroid lineage and is involved in carrying some blood group antigens. ${ }^{24}$ This molecule is a very specific marker of the erythroid line- age $^{18,26}$ and, in combination with CD71, as well as others molecules, is useful to study the maturation of the erythroid lineage, since its expression characterizes intermediate and late stages of normal erythroid maturation. ${ }^{18,26}$ In addition, Glycophorin-A is a very important diagnostic marker of acute erythroid leukemia, ${ }^{12,15,27,28}$ being positive in many cases.

In our case, a high percentage of monoblasts were positive for GlycophorinA and, to the best of our knowledge and after an extensive search of the literature available in PubMed archives, its positivity in AMoL has never been described previously.

We hypothesize that Glycophorin-A positivity in a case of $\mathrm{AMoL}$ could be explained as derivation of blast cells from a common myelo-erythroid precursor. In fact, according to the myeloid-based model of myelopoiesis, myeloid cells represent a prototype of blood cells, whereas erythroid, T and $\mathrm{B}$ cells represent specialized types. ${ }^{29}$ Thus, a common myelo-erythroid precursor is likely to derive from hematopoietic stem cells. ${ }^{17,29}$ undergoing subsequent separate differentiation into myeloid precursor cells and erythroid precursor cells. According to such a model, in our case the leukemogenic events could have interested a common myelo-erythroid precursor soon before the production of committed cells with different differentiation properties. Such a hypothesis could explain the permanence of
Glycophorin-A on the majority of leukemic cells belonging to the monocytic lineage.

Interestingly, according to the model proposed by Mori et al., ${ }^{17}$ the common myeloid-erythroid precursor might develop towards the erythroid-megakaryocytic lineage after up-regulation of CD71 and towards the myeloid lineage if CD71 remains down-regulated.

Finally, the co-existence of an erythroid molecule with myelo-monocytic markers might represent a very powerful leukemiaassociated immunophenotype, useful to assess response to therapy and to evaluate minimal residual disease.

\section{References}

1. Arber DA, Orazi A, Hasserjian R, et al. The 2016 revision to the World Health Organization classification of myeloid neoplasms and acute leukemia. Blood 2016;127:2391-405.

2. Tallman MS, Kim HT, Paietta E, et al. Acute monocytic leukemia (FrenchAmerican-British classification M5) does not have a worse prognosis than other subtypes of acute myeloid leukemia: a report from the Eastern Cooperative Oncology Group. J Clin Oncol 2004;22:1276-86.

3. Villeneuve P, Kim DT, Xu W, et al. The morphological subcategories of acute 
monocytic leukemia (M5a and M5b) share similar immunophenotypic and cytogenetic features and clinical outcomes. Leuk Res 2008;32: 269-73.

4. Bain BJ, Clark DM, Wilkins BS. Acute myeloid leukemia, mixed phenotype leukemia, the myelodysplastic syndromes and histiocytic neoplasms. In: Bain BJ, Clark D, Wilkins B, eds. Bone marrow pathology. Chichester, UK: Wiley-Blackwell; 2010. pp 172-175.

5. Pourabdollah M, Chang H. Plasma cell neoplasm with plasmablastic morphology mimicking acute leukemia. Blood 2017;129:132.

6. Moore EM, Roth CG. Plasma cell leukemia mimicking acute myeloid leukemia. Blood 2016;127:2359.

7. Gatter K, Brown D. Mature B cell neoplasms. In: Gatter K, Brown D, eds. Bone marrow diagnosis. An illustrated guide. Chichester, UK: Wiley Science Ltd; 2014. p 145.

8. Goasguen JE, Bennett JM, Bain BJ, et al. Morphological evaluation of monocytes and their precursors. Haematologica 2009;94:994-7.

9. Dunphy CH, Orton SO, Mantell J. et al. Relative contributions of enzyme cytochemistry and flow cytometric immunophenotyping to the evaluation of acute myeloid leukemias with a monocytic component and of flow cytometric immunophenotyping to the evaluation of absolute monocytoses. Am J Clin Pathol 2004;122:865-74.

10. Arber DA, Borowitz MJ, Cessna M, et al. Initial diagnostic workup of acute leukemia: guideline from the College of American Pathologists and the American Society of Hematology. Arch Pathol Lab Med 2017;141:1342-93.

11. Dunphy $\mathrm{CH}$, Tang W. The value of CD64 expression in distinguishing acute myeloid leukemia with monocytic differentiation from other subtypes of acute myeloid leukemia: a flow cyto- metric analysis of 64 cases. Arch Pathol Lab Med 2007;131:748-54.

12. Craig FE, Foon KA. Flow cytometric immunophenotyping for hematologic neoplasms. Blood 2008;111:3941-67.

13. Delgado J, Morado M, Jimenez MC, et al. CD56 expression in myeloperoxidase-negative

FAB M5 acute myeloid leukemia Am J Hematol 2002;69:28-30.

14. Miwa H, Mizutani M, Mahmud N, et al. Biphasic expression of CD4 in acute myelocytic leukemia (AML) cells: AML of monocyte origin and hematopoietic precursor cell origin. Leukemia 1998;12:44-51.

15. Peters JM, Ansari MQ. Multiparameter flow cytometry in the diagnosis and management of acute leukemia. Arch Pathol Lab Med 2011;135:44-54.

16. Zushi Y, Sasaki M, Mori A, et al. Acute monocytic leukemia diagnosed by flow cytometry includes acute myeloid leukemias with weakly or faintly positive non-specific esterase staining. Hematol Rep 2018;10:7435.

17. Mori Y, Che JY, Pluvinage JV, et al. Prospective isolation of human erythroid lineage-committed progenitors. Proc Natl Acad Sci U S A 2015;112:9638-43.

18. van Lochem EG, van der Velden VH, Wind HK, et al. Immunophenotypic differentiation patterns of normal hematopoiesis in human bone marrow: reference patterns for age-related changes and disease-induced shifts. Cytometry B Clin Cytom 2004;60:1-13.

19. Wu B, Shi N, Sun L, Liu L. Clinical value of high expression level of CD71 in acute myeloid leukemia. Neoplasma 2016;63:809-815.

20. Pande A, Dorwal P, Jain D, et al. Expression of CD71 by flow cytometry in acute leukemias: More often seen in acute myeloid leukemia. Indian $\mathrm{J}$ Pathol Microbiol 2016;59:310-3.
21. Liu Q, Wang $\mathrm{M}, \mathrm{Hu} \mathrm{Y}$, et al. Significance of CD71 expression by flow cytometry in diagnosis of acute leukemia. Leuk Lymphoma 2014;55:892-8.

22. El-Sakhawy YN, Pessar SA, Boshnak NH. Evaluation of CD71 Expression in Acute Leukemia in Egyptian Patients. JMSCR 2016;4:13147-56.

23. Bradstock K, Matthews J, Benson E, et al. Prognostic value of immunophenotyping in acute myeloid leukemia. Australian Leukaemia Study Group. Blood 1994;15:1220-5.

24. Kollia P, Stavroyianni N, Stamatopoulos K, et al. Molecular analysis of transferrin receptor mRNA expression in acute myeloid leukaemia. Br J Haematol 2001;115:1924.

25. Aoki T. A Comprehensive review of our current understanding of red blood cell (RBC) glycoproteins. Membranes 2017; 7:E56.

26. Mao B, Huang S, Lu X, et al. Early Development of Definitive Erythroblasts from Human Pluripotent Stem Cells Defined by Expression of Glycophorin A/CD235a, CD34, and CD36. Stem Cell Reports 2016;7:86983.

27. Braylan RC, Orfao A, Borowitz MJ Davis BH. Optimal number of reagents required to evaluate hematolymphoid neoplasias: results of an international consensus meeting. Cytometry 2001;46:23-7.

28. Zuo Z, Polski JM, Kasyan A, Medeiros LJ. Acute erythroid leukemia. Arch Pathol Lab Med 2010;134:1261-70.

29. Kawamoto H, Wada H, Katsura Y. A revised scheme for developmental pathways of hematopoietic cells: the myeloid-based model. Int Immunol 2010;22:65-70. 